\title{
The Remote and Centralized Control System for Wind Power Based on IEC 61400-25
}

\author{
Xiaoyun Ding, Liping Lu, Juan Liu \\ State Grid Electric Power Research Institute, Beijing SG-EPRI UHV Transmission Technology \\ Company, Beijing, 102200, China
}

Keywords: remote and centralized control system for wind power, IEC61400-25, regional control center.

\begin{abstract}
The vigorous development of wind power is the driving force behind several different monitoring systems for wind farms, thus arising an increasingly prominent problem of non-uniform communication protocols. The remote and centralized control system for wind power based on IEC 61400-25 can help standardize the wind power communication protocols and monitor different suppliers' information simultaneously, thus fulfilling the functions of data acquisition, device control, measurement, parameter adjustment and accident alarm, prediction and so on. At the same time, the system provides data interfaces so that it can operate coordinately with the higher level control center. The remote and centralized control system for wind power greatly reduces the number of field workers and improves intensive management.
\end{abstract}

\section{Introduction}

Since wind power is plentiful, clean, renewable and widely distributed, it attracts more and more attention from the countries and regions all over the world [1].

The utilization of wind power starts earlier abroad and large scale projects foster the development of Supervisory Control and Data Acquisition (SCADA) system. More details are in reference (2). There are still many problems in terms of monitoring and control management in wind farms. First of all, wind turbine suppliers' SCADA systems are mainly based on foreign systems and provide different communication protocols, mainly including OPC, Modbus or the ones from original equipment manufacturers. Different ways of connections and communication protocols coexist because the sensors and actuators are offered by various suppliers. What's more, wind farms are constructed by stages, leading to several different SCADA systems coexist. All these add difficulties to the operation management in wind farms [2].

A remote and centralized control system for wind farms is, therefore, urgent to be established. The system based on IEC 61400-25, an application of IEC 61850 in wind power, is designed to monitor and control wind farms. Based on open and standard internet technology, it is aimed at achieving communication among devices from different suppliers. Through a standard communication protocol, a common platform can achieve centralized monitoring and control of information of different generator systems in wind farms. The systematic analysis of the overall data about wind turbines will help control individual turbine precisely. The remote monitoring and control system can reduce the number of field workers.

\section{Remote and Centralized Control System for Wind Power}

\subsection{Hierarchical Network Architecture}

The central control center is established in the headquarters. Some provinces set up regional control centers, managing every wind farm within the region. Every wind farm builds the wind farm control center.

The four-layer system consists of collecting, wind farm control, regional control and central control layers [3]. 
1) Collecting Layer

Collecting layer is mainly responsible for collecting information of various types of devices in wind farms, including wind turbine control PLC and sensors, to keep track of wind turbines' data and states. It can start, stop, redirect and brake wind turbines and open oil pumps etc. In doing so, the individual wind turbine can be operated automatically.

2) Wind Farm Control Layer

Wind farm control layer is designed to integrate systems and data in the whole wind farm, including wind turbine suppliers' SCADA systems, power prediction systems, integrated automation systems, box transformer substation systems and five prevention systems. It also transmits the real time information of wind farms to the regional control layer.

Wind farm control layer uses windows system to adapt to some low-end communication protocols. With the upgrade of suppliers' protocols, the system can evolve to Linux system.

3) Centralized Control Layer

Communicating with wind farm control layer, centralized control layer is responsible for receiving operation information from all wind farms in the region and sending commands to wind farm control layers. Then the wind farm control layer sends commands to devices (wind turbines, booster stations etc.). It also transmits important real time information of wind farms in the region to central control layer.

4) Central Control Center

Central control layer accounts for integrated control and dispatch of all our wind farms scattered in the country.

Central control layer is designed to receive and analyze a sea of real time and historical data, thus providing optimal decisions for wind farms and instructive suggestions for intensive management of wind power. The conventional system architecture cannot handle such amount of information, so the new system should adopt system based on cloud computing [4].

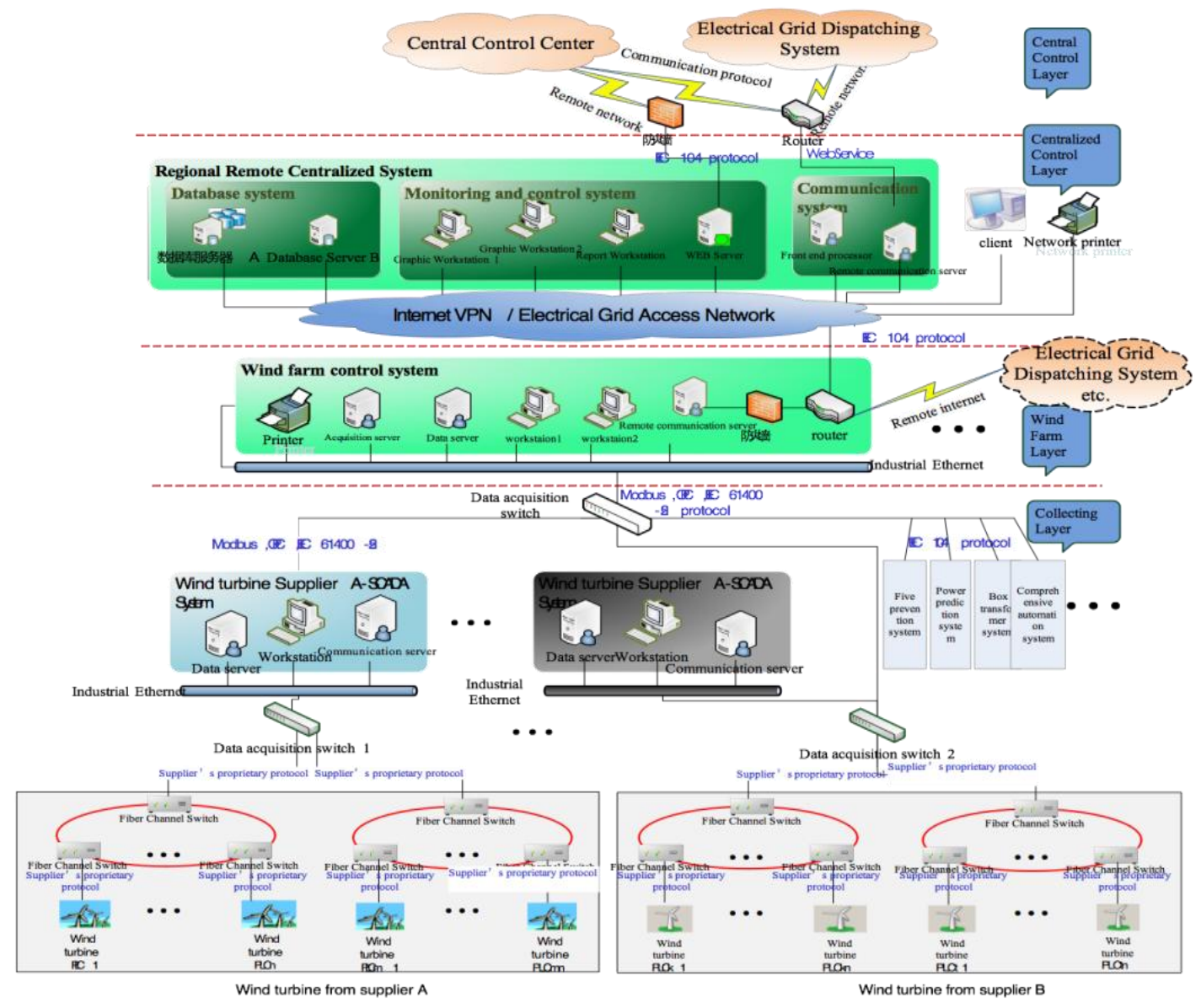

Fig. 1 The overall system architecture diagram 


\subsection{Overall System Architecture}

Given the realization of top-down centralized management and control, three systems should be established, namely wind farm control system, regional remote and centralized control system and central control system in the company's headquarters. The remote and centralized control system for wind power is designed in regional control center. It can handle 500,000 points of data capacity [5-7] at best. The overall system architecture is shown in Fig. 1.

\subsection{Hardware Components}

Given the scale of wind farm and data volume, a wind farm can be equipped with hardware devices accordingly. For the smallest wind farm, it can be equipped with only one server to fulfill all functions. The whole system requires the following hardware devices and servers, front end processors, data servers, application servers, remote communication servers, WEB servers, hardware firewalls and switches.

\section{Communication Solutions}

The communication interfaces and protocols of supplies' wind turbine controllers and SCADA system are incompatible with each other. In addition, suppliers' communication protocols are not open to each other. In order to remove communication barriers, the article adopts the unified communication protocol standard for wind farms and develops the unified communication interface [8].

\subsection{Unified Communication Protocol}

IEC 61400-25 extends the technology of the IEC 61850 standard to address needs particular to wind power [9]. Based on open and standard internet technology, it is aimed at achieving communication among devices from different suppliers. The remote and control system for wind power can send instructions simultaneously to different wind turbines and coordinate their operation.

\subsection{Standardized Modeling}

Based on IEC61400-25 standard and referring to related wind turbine controller (YOKOGAWA, Bachmann), the system designs the wind turbine model, which includes tower, nacelle, generator, transformer, variable-frequency drive, hub, rotor, blade, transmission system and yaw system. And then it establishes tables of wind farms and wind turbine parameter. But the site monitoring and control systems from different suppliers provide various parameters, so the point table must be provided on the basis of the whole input and output. According to foreign key association, related wind turbines establish other systems' user table and role table and user permission table to manage user permission.

\subsection{Standardized Communication Interfaces}

Wind farms are distributed energy resources systems. With a sea of data, they scatter in poor environment. The communication system must be capable of transmitting a sea of real time and reliable data.

Communication between Wind Turbine Controller and supplier's SCADA System.

At present, wind farms are equipped with large-scale grid-connected wind turbine generator systems, of which each has own control system (mainly including PLC controllers). The wind turbine supplier's SCADA system can communicate with several controllers simultaneously. Connected by a switch and optical fiber ring network and adopt MODBUS(TCP/IP) protocol, OPC protocol and suppliers' private protocols, SCADA system and controllers cannot exchange standardized information. The private protocols, equipped by most suppliers' wind turbine controllers, will be replaced by IEC61400-25 protocol [10].

SCADA system can exchange data with every generator system through generator systems' own communication interfaces (achieved by PLC) [11, 12].

Communication between Supplier's SCADA system and Wind Farm Control Systems.

In terms of communicating with wind farm control centers, SCADA system will replace MODBUS (TCP/IP) protocol and OPC protocol with IEC61400-25.

Communication between Wind Farm Control Systems and Regional Remote and Centralized Control Systems. 
Adopting 100/1000M auto-adaptive dual Ethernet structure, two industrial Ethernet switches can connect all devices, making the system more reliable. Since Ethernet is connected to the Internet, the real time data and parameters of wind farms can be sent to regional control centers. IEC104 protocol or Webservice interfaces are recommended to communicate with other systems, such as ERP system or dispatching systems.

\section{Conclusion}

The remote and centralized control system for wind power based on IEC61400-25 can integrate all systems and collect data together. This article designs the overall system architecture, analyzes its functions on different layers, introduces the hardware components and software functions and proposes communication solutions. So this system will help reduce the number of filed workers and improve intense management. In addition, given that the variability of intermittent wind power resources leads to the unstable power grid, this system can coordinate the operation of all wind farms in the region and control individual wind turbines effectively so that the economic benefits of the power system in the whole region.

\section{References}

[1] YANG Wen-hua. Summary of current situation and development trend of the wind farm monitoring system[J]. Ningxia Electric Power,2011,(4):51-56.

[2] QING Chang-gui. SCADA system and its application in wind farm[J]. Electric Equipment, 2004, $5(12): 31-33$.

[3] SONG Xiao-ping, LIAO Ming-fu. Wind farm SCADA System Framework Based on Internet[J]. Automation of Electric Power Systems,2006,30(17):89-93.

[4] YE Jian-bing, ZUO Jian-fei,et al. The design of Wind farm cluster's remote centralized SCADA system [J]. Automation of Electric Power Systems,2010,34(23):97-101.

[5] ZHOU Quan-ren, ZHANG Hai-tao.Discussion of the design for Monitoring and Control system for wind power.[M].Beijing:China Electric Power Press,2004,133-139.

[6] LONG Xun,CAI Jian-yun. The development of wind farm remote monitoring system based on configuration software[J]. Energy and Environment, 2007 (2) :76-78.

[7] DUAN Bin, ZOU Ji-chang, SU Yong-xin, et al. Design and application Wind farm monitoring software integration services model[J]. Automation of Electric Power Systems, 2008, 32 (10): 88-93.

[8] HE Tao, LI Wei. Wind farm monitoring communication standard IEC 61400-25 and design ideas[J]. System And Clean Energy,2009,25 (8) : 45-49.

[9] IEC61400-25-2.Communication for monitoring and control of wind power plants-overall description of principles and models [S].2006.

[10] ZHU Bing-quan, REN Yan-ming, JING Jian-ning, et al. Substation automation system to achieve the transitional period of the policy IEC 61850[J].Automation of Electric Power Systems, 2005, 29 (23): 54-57.

[11] FAN Jian-zhong, MU Lian-shun, WANG Hai-ling. Data modeling for substation SCADA system based on IEC61850 standard[J].Automation of Electric Power Systems, 200630(5):43-48.

[12] YANG Da-quan, BAO Jin-yan, XINg Zuo-xia, WANG Xiao-dong, HOU Jun. Data transmission technology of SCADA system in wind power plant [J]. Journal of Shenyang University of Technology, 2008, 30(6):632-638. 